Natalia Rekowska*, Jan Konasch, Alexander Riess, Robert Mau, Thomas Eickner, Hermann Seitz, Niels Grabow and Michael Teske

\title{
PEGDA drug delivery scaffolds prepared with UV curing process
}

\begin{abstract}
Individually tailored drug delivery systems (DDSs) are considered one of the most promising therapeutic tools for the creation of safe and effective treatments. DDSs as a novel approach should be beneficial in curing systemic as well as local ailments, where a high topical concentration of the drug and a reduction of side effects are desirable. It could also be favorable for patients requiring customized treatments, showing atypical profiles of drug metabolism. Development of particular drug delivery devices require the selection of a suitable scaffold material, which should exhibit proper mechanical and biological properties, but also enable adjustment of the drug release according to a specific need. Thus, it is extremely important to expand the knowledge concerning potential DDS components. Poly(ethylene glycol) diacrylate (PEGDA) according to its properties can be easily used as a DDS resin and shaped into a desired structure with the employment of techniques based on photopolymerization, including some novel $3 \mathrm{D}$ printing techniques. As a continuation of our previous works, in this paper drug release studies from conventionally prepared PEGDA scaffolds are presented. We have shown that in PEGDA materials, the release profile of the low molecular weight model drug acetylsalicylic acid can be altered by water content. PEGDA as a delivery material should be further investigated to specify its potential as a comonomer and a matrix for pharmaceutical agents.
\end{abstract}

Keywords: Drug delivery system (DDS), Poly(ethylene glycol) diacrylate (PEGDA), acetylsalicylic acid (ASA) release.

https://doi.org/10.1515/cdbme-2020-3049

\footnotetext{
${ }^{*}$ Corresponding author: Natalia Rekowska, Institute for Biomedical Engineering, University Medical Center Rostock, Friedrich-Barnewitz-Straße 4, 18119 Rostock, Germany, e-mail: natalia.rekowska@uni-rostock.de Thomas Eickner, Niels Grabow, Michael Teske: Institute for Biomedical Engineering, University Medical Center Rostock, Germany.

Jan Konasch, Alexander Riess, Robert Mau, Hermann Seitz: University of Rostock, Chair of Microfluidics, Rostock, Germany.
}

\section{Introduction}

The improvement of diagnostic tools provided a deep understanding of the disposition of pharmaceutical compounds in the human body. It also revealed a serious issue concerning pharmacotherapy: the individual variability [1]. The demand of designing treatments tailored to patients' needs applies particularly to such groups as elderly and pediatric populations, polymedicated patients and those suffering from metabolic diseases [2,3]

Recent advances in pharmaceutical technology enable designing increasingly safer and more effective medicinal therapies for particular individuals. Among novel approaches, drug delivery systems (DDSs) are considered especially beneficial according to many advantages over traditional routes of administration [4]. The technology of DDSs is being continuously developed to obtain the best therapeutic effect for systemic or local treatments with marginal side effects. Different materials, in particular synthetic polymers, are investigated as scaffold components to form a device releasing an active substance in a desired manner.

One of frequently used material for this purpose is poly(ethylene glycol) diacrylate (PEGDA) - a photopolymerisable compound, which is considered as nontoxic, cytocompatible and easy to use [5]. It can be employed in fabrication of drug delivery scaffolds of different shapes and applications. Furthermore, its potential as a DDS resin has been confirmed in numerous works involving both simple and more advanced, innovative 3D manufacturing techniques $[6,7]$.

In our previous investigations we have analyzed the properties of PEGDA as a pure and copolymerized material with a few other acrylates $[8,9]$. These studies have shown the influence of different factors such as washing procedure, copolymer composition and photoinitiator (PI) concentration on the thermomechanical and biological properties of the samples. Here, we present the results of drug release studies with the employment of a model drug, acetylsalicylic acid (ASA) from UV polymerized samples for the pure PEGDA samples and the samples with increased water content. 


\section{Materials and methods}

\subsection{Chemicals}

Poly(ethyleneglycol) diacrylate $\quad \mathrm{M}_{\mathrm{n}}=700 \mathrm{~g} / \mathrm{mol}$ (PEGDA), PI: Lithium phenyl-2,4,6-trimethylbenzoylphosphinate (LAP), PI solvent methanol, acetylsalicylic acid and its solvent dimethyl sulfoxide were purchased from Merck KGaA (Darmstadt, Germany). The ultrapure water required for obtaining $\mathrm{PEGDA} / \mathrm{H}_{2} \mathrm{O}$ samples was obtained from the arium ${ }^{\circledR}$ purifying device (Sartorius $\mathrm{GmbH}$, Göttingen, Germany).

\subsection{Sample preparation}

Pure PEGDA or $50 \%$ v/v PEGDA solutions, diluted in ultrapure water, were prepared. ASA solution in DMSO was prepared and added to PEGDA solution to obtain samples containing different amounts of ASA (20, 10, 5 and $2.5 \mu \mathrm{g})$. Reference samples without the addition of ASA were also prepared. Samples were polymerized as specified in our previous work [8] in a silicone holder (to obtain sample design according to the ISO 527-2) in the UV chamber at $\lambda=365 \mathrm{~nm}$ for 10 minutes.

\subsection{Drug release studies}

Each sample was immersed in $4 \mathrm{~mL}$ of isotonic sodium chloride solution and underwent elution at $37^{\circ} \mathrm{C}, 100 \mathrm{rpm}$ and in protection from light. The release studies were continued for $48 \mathrm{~h}$, with the complete medium change at particular time points. Afterwards the complete residual release of ASA was performed in methanol.

\subsection{ASA determination}

The released amounts of ASA were determined with a HPLC device (Wiss. Gerätebau Dr.-Ing. H. Knauer GmbH, Germany). C18 TL76 $5 \mu \mathrm{m}, 24 \times 4 \mathrm{~mm}$ column was used. Isocratic elution with the use of water/ACN/ $\mathrm{H}_{3} \mathrm{PO}_{4}$ (600:400:2) was performed. Standard solutions for HPLC calibration were prepared in methanol.

\section{Results}

\subsection{ASA release}

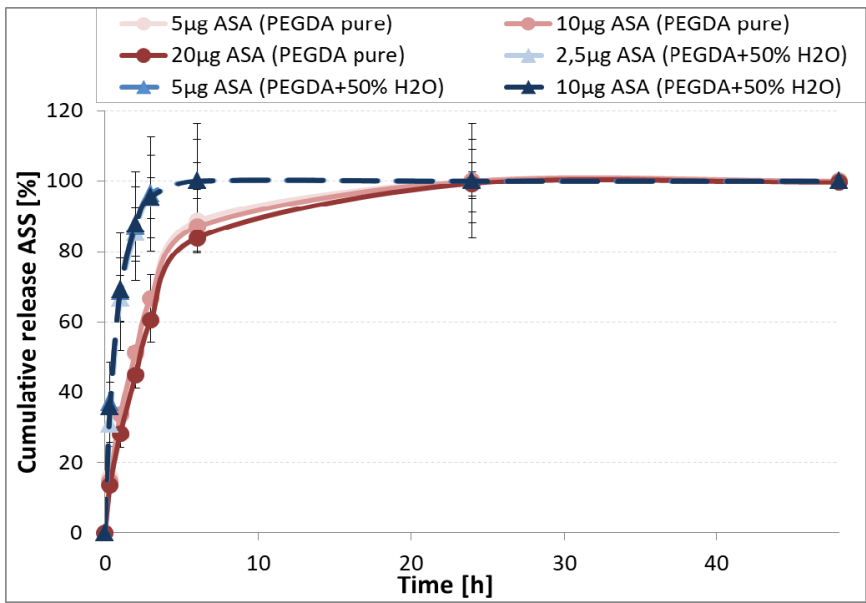

Figure 1: The relative cumulative release of ASA in [\%] within 48 hours from PEGDA pure (red) and PEGDA $+50 \% \mathrm{H}_{2} \mathrm{O}$ (blue) samples containing different amounts of ASA. Release in $4 \mathrm{~mL}$ of $0.9 \% \mathrm{NaCl}$ at $37^{\circ} \mathrm{C}$ at $100 \mathrm{rpm}(\mathrm{n}=3)$.

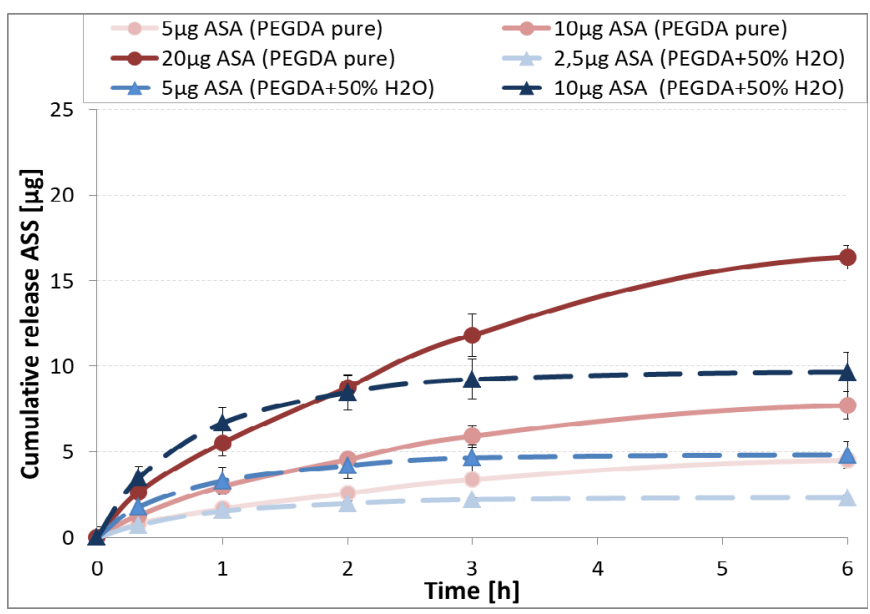

Figure 2: The first 6 hours of absolute cumulative release of ASA in $[\mu \mathrm{g}]$ from pure PEGDA (red) and PEGDA $+50 \%$ $\mathrm{H}_{2} \mathrm{O}$ (blue) samples containing different amounts of ASA, Release in $4 \mathrm{~mL}$ of $0.9 \% \mathrm{NaCl}$ at $37^{\circ} \mathrm{C}$ at $100 \mathrm{rpm}(\mathrm{n}=3)$.

Figure 1 illustrates the relative release values of ASA from samples containing different amounts of ASA as a model drug. Some of the samples were prepared with the addition of $50 \% \mathrm{v} / \mathrm{v}$ of water. In the case of these samples, after two hours $82 \%$ on average of ASA was already released, compared to pure PEGDA samples where at the same time point $42 \%$ of incorporated ASA were released. Here, the release of $83 \%$ of the ASA was observed after 6 hours. After 24 hours in both types of samples the ASA 
release was completed and no residual ASA was observed in the samples. The differences between results of the samples containing different amounts of ASA are mostly minor and are more distinct in pure PEGDA samples.

In Figure 2 the first 6 hours of the absolute cumulative release in $[\mu \mathrm{g}]$ are shown. After 2 hours of the release the PEGDA pure sample containing $20 \mu \mathrm{g}$ of ASA released 8.76 $\mu \mathrm{g}(43.8 \%)$ of the agent, while in case of corresponding PEGDA $+50 \% \mathrm{H}_{2} \mathrm{O}$ sample containing $10 \mu \mathrm{g}$ of ASA (same ASA concentration in sample) it is $8.47 \mu \mathrm{g}(84.7 \%)$. After 3 hours the average release of ASA in all pure PEGDA samples reached $62 \%$ and in all PEGDA $+50 \%$ water samples $91 \%$.

\section{Discussion}

The factors influencing the drug release rate are one of the most important and interesting aspects in the development of drug delivery materials.

The drug release curves of PEGDA pure and PEGDA + $50 \% \mathrm{H}_{2} \mathrm{O}$ samples illustrated above showed the existing differences between these two drug delivery systems for the low molecular weight model active substance - ASA. The slope of ASA release in PEGDA $+\mathrm{H}_{2} \mathrm{O}$ samples is steeper in comparison with their corresponding PEGDA pure samples and the release is finished rapidly. Therefore we observe a much stronger burst release resulting in an earlier release and a much higher amount of released ASA. The possible explanation of this phenomenon can be explained by the fact, that the water dilutes the polymer and therefore the crosslinking density decreases, the pores in the scaffold are probably larger, which facilitate water penetration and diffusion of the drug to the release medium. According to the lack of functional groups of ASA, which could be activated by the PI to form covalent bonds with PEGDA, the release is only dependent on the diffusion rate.

\section{Conclusion}

The results presented in this paper characterise PEGDA as a drug delivery material. It was shown that the drug release rate of the model drug ASA can be influenced by polymer concentration and scaffold structuring via water dilution.

Further studies concerning PEGDA copolymerised with other acrylates and different model drugs, for example proteins should be conducted to broaden the knowledge concerning this photopolymer.

\section{Author Statement}

Research funding: Financial support by German Research Foundation (DFG) within the project "3D printed drug delivery systems with the ability of time controlled drug release" (TE1233/1-1) is gratefully acknowledged.

Conflict of interest: The authors state no conflict of interest.

Ethical approval: The conducted research is not related to either human or animal use.

\section{References}

[1] S. Kotta, A. Nair and N. Alsabeelah, "3D Printing Technology in Drug Delivery: Recent Progress and Application" Current pharmaceutical design, vol. 24, no. 42, pp. 5039-5048, 2018.

[2] N. Sandler and M. Preis, "Printed Drug-Delivery Systems for Improved Patient Treatment," Trends in pharmacological sciences, vol. 37, no. 12, pp. 10701080, 2016.

[3] C. Li, J. Wang, Y. Wang et al., "Recent progress in drug delivery," Acta pharmaceutica Sinica. B, vol. 9, no. 6, pp. 1145-1162, 2019.

[4] G. Tiwari, R. Tiwari, B. Sriwastawa et al., "Drug delivery systems: An updated review," International journal of pharmaceutical investigation, vol. 2, no. 1, pp. 2-11, 2012.

[5] J. P. Mazzoccoli, D. L. Feke, H. Baskaran et al., "Mechanical and cell viability properties of crosslinked low- and high-molecular weight poly(ethylene glycol) diacrylate blends," Journal of biomedical materials research. Part A, vol. 93, no. 2, pp. 558-566, 2010. [6] J. Konasch, A. Riess, A.; H. Seitz et al., "A Novel Hybrid Additive Manufacturing Process for Drug Delivery Systems with Locally Incorporated Drug Depots," Pharmaceutics, 11, 661, 2019.

[7] J. R. Choi, K. W. Yong, J. Y. Choi et al., "Recent advances in photo-crosslinkable hydrogels for biomedical applications," BioTechniques, vol. 66, no. 1, pp. 40-53, 2019.

[8] N. Rekowska, D. Arbeiter, T. Eickner et al., "Thermomechanical properties of PEGDA in combination with different photo-curable comonomers" CDBE, vol. 5, no. 1, pp. 319-321, 2019.

[9] N. Rekowska, D. Arbeiter, J. Konasch et al., "Thermomechanical properties of PEGDA and its copolymers," CDBE, vol. 4, no. 1, pp. 669-672, 2018. 